\title{
Mitral valve myxoma associated with intracranial tumor: a case report
}

\author{
Arif Gucu, Deniz Demir, Nail Kahraman, Mesut Engin, Ahmet Fatih Ozyazicioglu, Mehmet Tugrul Goncu
}

Department of Cardiovascular Surgery, Bursa Yuksek Ihtisas Training and Research Hospital, Bursa, Turkey

\begin{abstract}
Primary tumors of the heart are rare, and among them, cardiac myxoma is the most prevalent primary cardiac neoplasm in adult patients. Astrocytoma is the most common glioma and can occur in most parts of the brain and occasionally in the spinal cord. There is a little knowledge about coexistence of cardiac myxoma and astrocytoma in the literature. Cardiac myxoma associated with intracranial tumor is a very rare entity. We presented a case of cardiac myxoma originating mitral anterior valve associated with astrocytoma. The patient underwent the operation for intracranial tumor 2 months before cardiac surgery. Mitral valve myxoma was successfully treated with surgical resection without mitral valve replacement.
\end{abstract}

Eur Res $J$ 2016;2(3):233-235

Keywords: Cardiac myxoma; mitral valve myxoma; intracranial tumor; astrocytoma

\section{Introduction}

Primary tumors of the heart are rare, and cardiac myxoma represents the most common type of primary cardiac tumor. About $80 \%$ of primary cardiac tumors are benign, and of these more than half are myxomas. Myxomas occur in all age groups, but are especially frequent 30-60 years, with a female predilection [1]. About $75 \%$ of these tumors arise from the left atrium and $20-25 \%$ from the right atrium. Tumors originate from atypical sites such as left and right ventricle and valves are very rare, and have occasionally been found in all four cardiac chambers [2]. Astrocytomas are a type of cancer of the brain. They originate in a particular kind of glial cells, star-shaped brain cells in the cerebrum called astrocytes. Association of astrocytoma and cardiac myxoma is very rare.

\section{Case Presentation}

A 66-year-old man was admitted to our hospital for a routine cardiac examination. In past medical history, he had undergone surgery for intracranial tumor 2 months ago. Pathological analysis of this tumor was consistent with low-grade, World Health Organization grade II astrocytoma.

He had no history of cardiac symptoms, syncope or fever. On examination vital signs were as follows: blood pressure 120/80 $\mathrm{mmHg}$, pulse 76 beats/min. Blood biochemistry was revealed to be normal. The electrocardiogram demonstrated regular sinus rhythm and the chest $\mathrm{x}$-ray was normal. Transthoracic echocardiography revealed a mobile mass, $31 \times 20 \mathrm{~mm}$, attached to the ventricular surface of the anterior mitral leaflet. There was mild mitral regurgitation. The 
left ventricular function was normal.

The patient was scheduled for cardiac surgery. Standard aortic and bi-caval cannulation was performed. Myocardial protection was provided by means of antegrade cardioplegic solution with topical and mild systemic cooling. The left atrium was approached through the interatrial groove. Myxoma was attached to the atrial surface of the anterior mitral leaflet. Appearance of this tumor was yellowish translucent jelly and shiny (Figure 1a). Tumor was excised preserving the structure of mitral valve (Figure 1b). After excision, minimal mitral regurgitation was observed. On pathological examination, tumor was identified as myxoma.

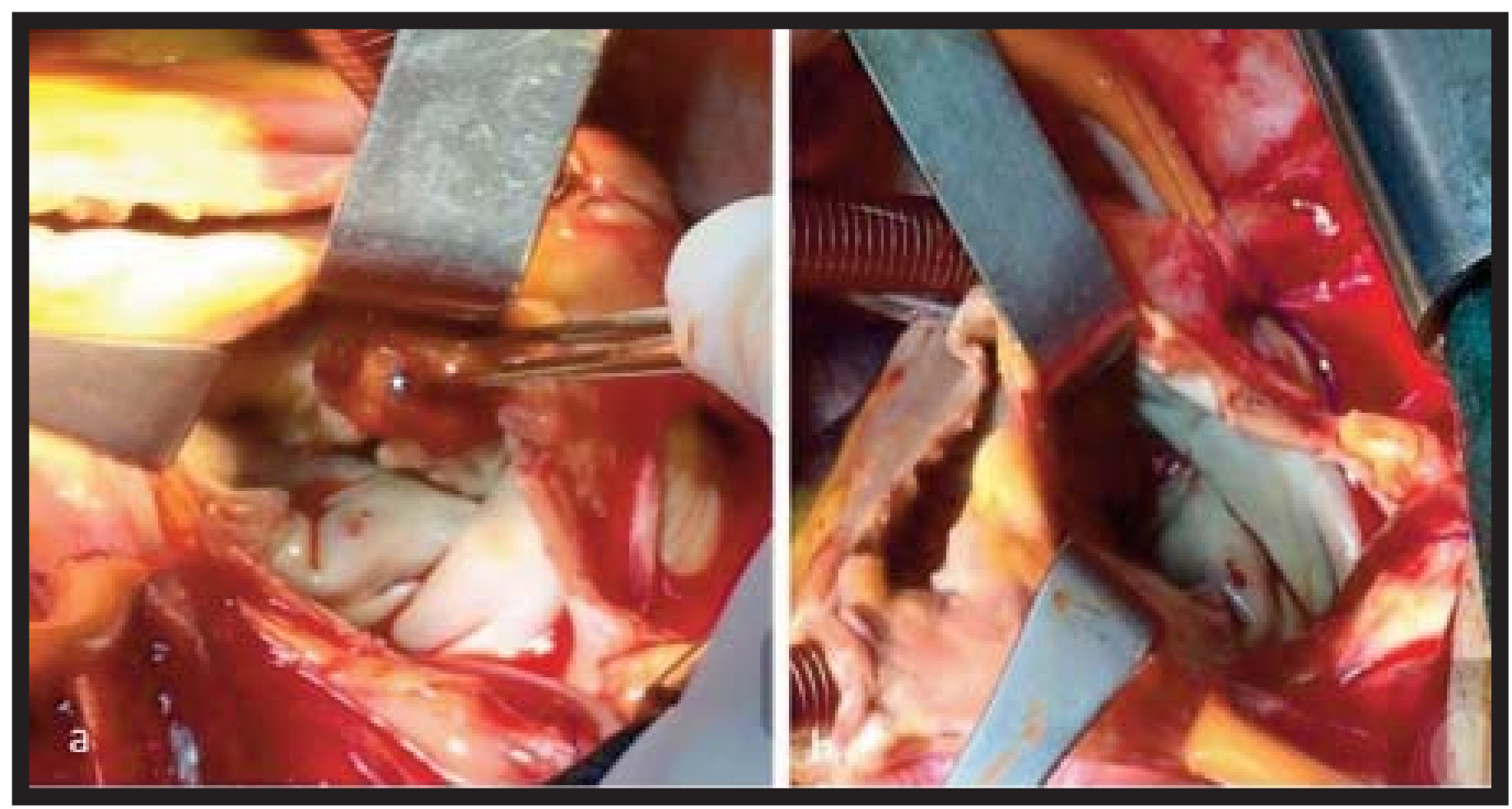

Figure 1. (a) Intraoperative appearance of the myxoma (asterisk). (b) The myxoma was excised preserving the structure of mitral valve.

\section{Discussion}

Myxoma is the most prevalent primary cardiac tumor. Myxomas can be originated in atypical locations, arising from the posterior or anterior left atrial wall, and arising from the mitral valve. Mitral valve myxomas is very rare. When the mitral valve is involved, the myxoma is frequently located on the atrial side with equal distribution between the anterior and the posterior leaflets [3-5]. The exact incidence of myxomas originating from the mitral valve is not clear.

Cardiac myxomas can be found along with other tumoral formation in the body. Complex cardiac myxomas are a classification of familial tumours, and occur as a syndromic presentation, requiring the presence of a cardiac myxoma with any two or more of the following concurrent conditions; skin myxomas, cutaneous lentiginosis, myxoid fibromas of the breast, pituitary adenoma, primary adrenocortical micronodular dysplasia with Cushing's syndrome, testicular tumours [6]. There are few reports in the literature about the association of glial tumors and cardiac myxoma [7]. This association is probably coincidental and there is no familial property.

Cardiac myxomas may restrict valve opening, causing functional mitral stenosis. In this patient, the tumor led to annular dilation and destruction of the valve, resulting in mitral insufficiency [8]. Clinical manifestations of myxoma are determined by the location, size, mobility, and friability, and can be divided into 3 general categories: systemic symptoms, embolism, and intracardiac obstruction. Echocardiography is most important diagnostic modality available for imaging cardiac tumors; it is noninvasive and does not pose the risk of tumor embolization. Echocardiography easily defines the size, location, shape, morphological characteristics, and relations of mass with intracardiac components $[9$, $10]$.

The manifestations of mitral valve myxoma may be cerebral or peripheral embolism. When located on 
the mitral valve, they usually occur on the atrial surface of the valve and the anterior and posterior leaflets are involved with equal frequency. The treatment of choice for myxoma is surgical removal, and complete excision is the goal. Immediate postoperative mortality ranges from $0 \%$ to $3.6 \%$ [10]. Arrhythmia is a common postoperative complication, which may require long-term medication.

All mitral valve myxomas require surgical resection because of their potential to obstruct the valve orifice, dilate the annulus, embolism, or cause rhythm disturbances. The debate for uniatrial and biatrial approach continues. Most authors think that a uni-atrial approach, especially for the left atrial myxoma is adequate [11]. However, a large series reported from Texas Heart Institute advocates an aggressive approach and that the bi-atrial approach may help the surgeon to handle the tissues more gently $[2,5,10-12]$. We used left atriotomy. Thinking of the benign nature of the myxomas, a more conservative approach may prevail.

Cardiac surgery in patients with malignant diseases remains a problem. Although most malignant diseases are curable, surgeons are usually reluctant to perform open heart surgery in patients with advanced tumors and a short life expectancy. Among patients undergoing open heart surgery, the incidence of malignancy is $1.2 \%$ [13]. Due to the risk of intracranial hemorrhage which our patient had previously intracranial tumor operation, we choose myxoma excision. The preferred surgical technique is excision without valve resection if possible. Resultant varying degrees of mitral valve insufficiency can be treated by primary or patch repair of the valve or replacement with a prosthesis [3]. Myxomas should be completely resected to avoid recurrence. In Garatti et al.'s [1] study, freedom from tumor recurrence was $92 \%, 91 \%$, and $86 \%$ at 10,20 , and 30 years, respectively. Younger age, smaller tumor dimension, and tumor localized to the ventricles were predictors of recurrence. It is not known whether replacement of mitral valve reduces the recurrence of mitral valve myxoma. Recurrences are usually managed by reexcision.

\section{Conclusion}

Although myxomas rarely can be seen with intracranial tumors, other possible concomitant intracranial tumors should be investigated in the presence of myxoma.

\section{Informed consent}

Written informed consent was obtained from the patient for the publication of this case report.

\section{Conflict of interest}

The authors declared that there are no potential conflicts of interest with respect to the research, authorship, and/or publication of this article.

\section{References}

[1] Garatti A, Nano G, Canziani A, Gagliardotto P, Mossuto E, Frigiola A, et al. Surgical excision of cardiac myxomas: twenty years' experience at a single institution. Ann Thorac Surg 2012;93:825-31.

[2] Samanidisa G, Perreasa K, Kalogrisa P, Dimitrioua S, Balanikab M, Amanatidisa G, et al. Surgical treatment of primary intracardiac myxoma: 19 years of experience. Interact Cardiovasc Thorac Surg 2011;13:597-600.

[3] Yavuz S, Celkan A, Ata Y, Mavi M, Turk, T, Eris C, et al. Mitral valve myxoma. Asian Cardiovasc Thorac Ann 2000;8: 646.

[4] Arruda MV, Braile DM, Joaquim MR, Soares MJ, Alves RH. Resection of left ventricular myxoma after embolic stroke. Rev Bras Cir Cardiovasc 2008;23:578-80.

[5] Choi BW, Ryu SJ, Chang BC, Choe KO. Myxoma attached to both atrial and ventricular sides of the mitral valve: report of a case and review of 31 cases of mitral myxoma. Int J Cardiovasc Imaging 2001;17:411-6.

[6] Raith EP. Heart: cardiac myxoma. Atlas Genet Cytogenet Oncol Haematol 2010;14:164-8.

[7] Chozick BS, Ambler MW, Stoll J Jr. Malignant astrocytoma six years after the resection of a cerebral metastatic cardiac myxoma: case report. Neurosurgery 1992;30:923-6; discussion 926-7.

[8] Kaya M, Ersoy B, Yeniterzi M. Mitral valve regurgitation due to annular dilatation caused by a huge and floating left atrial myxoma. Kardiochir Torakochirurgia Pol 2015;12:248-50.

[9] Lobo A, Lewis JF, Conti CR. Intracardiac masses detected by echocardiography: case presentations and review of the literature. Clin Cardiol 2000;23:702-8.

[10] Ipek G, Erentug V, Bozbuga N, Polat A, Guler M, Kirali K, et al. Surgical management of cardiac myxoma. J Card Surg 2005; 20:300-4.

[11] Seub BKH. Surgical considerations in the treatment of cardiac myxoma. J Thorac Cardiovasc Surg 1984;87:251-9.

[12] Yilman M, Kolbakir F, Keceligil HT, Keyik T, Erk MK. Myxoma originated from mitral annulus. Turk Gogus Kalp Dama 1994;2:159-61.

[13] Narin C, Ege E, Onoglu R, Yazici M, Sarigul A. Surgery of left atrial myxoma as a second primary tumor in a patient previously treated for breast cancer. Turk Gogus Kalp Dama 2012;20:133-5. 